
\title{
25 Research Square \\ Bidirectional Control of Coronary Vascular Resistance by Eicosanoids via a Novel GPCR
}

\section{Nabil Alkayed ( $\nabla$ alkayedn@ohsu.edu )}

Oregon Health \& Science University https://orcid.org/0000-0002-3489-4730

\section{Zhiping Cao}

Oregon Health \& Science University

\section{Zu Yuan Qian}

Oregon Health \& Science University

\section{Shanthi Nagarajan}

Oregon Health \& Science University

\section{Carmen Methner}

Oregon Health \& Science University

Xuehong Liu

Oregon Health \& Science University Jonathan Nelson

Oregon Health \& Science University

\section{Fuchun Xie}

Oregon Health \& Science University

Bingbing $\mathrm{Li}$

Oregon Health \& Science University

Wei Fan

Oregon Health \& Science University

Lijuan Liu

Oregon Health \& Science University

\section{Marjorie Grafe}

Oregon Health and Science

\section{Xiangshu Xiao}

Oregon Health \& Science University

\section{Anthony Barnes}

Oregon Health \& Science University

\section{Sanjiv Kaul}

Oregon Health \& Science University 
Keywords: GPR39, coronary vascular resistance, eicosanoids

Posted Date: June 2nd, 2021

DOI: https://doi.org/10.21203/rs.3.rs-506030/v1

License: (c) (1) This work is licensed under a Creative Commons Attribution 4.0 International License. Read Full License 


\section{Abstract}

Arachidonic acid metabolites epoxyeicosatrienoates (EETs) and hydroxyeicosatetraenoates (HETEs) are important regulators of myocardial blood flow and coronary vascular resistance (CVR), but their mechanisms of action are not fully understood. We identified G protein-coupled receptor 39 (GPR39) as a microvascular smooth muscle cell ( $\mathrm{mVSMC}$ ) receptor antagonistically regulated by two endogenous eicosanoids: 15-HETE, which stimulates GPR39 to increase mVSMC intracellular calcium and augment microvascular CVR, and 14,15-EET, which inhibits these actions. Furthermore, zinc ion acts as an allosteric modulator of GPR39 to potentiate the efficacy of the two ligands. Finally, GPR39 knockout mice are protected from myocardial ischemia compared to wild-type littermates. Our findings will have a major impact on understanding the roles of eicosanoids in cardiovascular physiology and disease, and provide an opportunity for the development of novel GPR39-targeting therapies for cardiovascular disease.

One Sentence Summary: GPR39 is a microvascular smooth muscle cell receptor regulated by two vasoactive eicosanoids with opposing actions.

\section{Main Text}

Myocardial oxygen demand determines coronary blood flow, which is exquisitely regulated through changing vascular resistance in coronary arterioles ranging in size from $100 \mu \mathrm{m}$ to $300 \mu \mathrm{m}$ (1). Whereas the determinants of myocardial oxygen demand are well known, the molecules responsible for the second-to-second regulation of microvascular tone and the receptors through which they act have not been identified. Two classes of signaling P450 eicosanoids, epoxyeicosatrienoates (EETs) and hydroxyeicosatetraenoates (HETEs), are potent regulators of microvascular tone and play critical roles in cardiovascular physiology and disease $(2,3)$. EETs are predominantly microvascular dilators and have been shown to function as endothelium-derived hyperpolarization factors (EDHFs) in the coronary circulation $(4,5)$, and to mediate functional hyperemia in the brain $(6,7)$. They are anti-inflammatory, inhibit platelet aggregation and protect against ischemia-reperfusion (I/R) injury in heart and brain (8). HETEs, on the other hand, are typically vasoconstrictors that have been implicated in the generation of arteriolar myogenic tone and blood flow autoregulation in the brain and kidneys $(9,10)$. They promote inflammation and contribute to hypertension and I/R injury (11).

The mechanisms utilized by these eicosanoids to exhibit their biological actions in microvascular smooth muscle cells (mVSMCs) remain largely unknown. Ligand binding and pharmacological studies suggest that EETs signal via a G protein-coupled receptor (GPCR) (12), yet the identity of an EETs receptor has remained elusive. In the current study, we generated a clickable photocrosslinking probe based on 14,15EET that allowed us to isolate its binding proteins from mouse heart mVSMCs in an unbiased manner using mass spectrometry-based proteomics. This approach identified GPR39, a member of the Ghrelin peptide receptor family, as a putative receptor for 14,15-EET in mVSMCs. Here, we show that GPR39 is capable of mobilizing calcium in VSMC in response to 15-HETE as well as serving as the site of 14,15EET's inhibitory action on 15-HETE-induced calcium signaling, indicating that it acts as a receptor for 
both eicosanoids. We also localize GPR39 immunoreactivity to mouse heart mVSMCs, and demonstrate 15-HETE increases CVR using the isolated Langendorff mouse heart perfusion preparation, an effect that is inhibited by co-administration of 14,15-EET and requires GPR39 expression. Finally, we demonstrate that deletion of GPR39 protects mice from myocardial I/R injury in vivo, suggesting that GPR39 may serve as a therapeutic target in ischemic heart disease.

\section{Results}

\section{Identification of GPR39 as a putative receptor for 14,15-EET}

We implemented a chemical biology approach to purify 14,15 -EET-binding proteins by producing a modified form of 14,15-EET (EET-P, Fig. 1A and Supplementary Material) that covalently crosslinks to target proteins following exposure to ultraviolet (UV) light. The probe also incorporates a click chemistry moiety that allows subsequent fluorophore labeling or biotin-streptavidin affinity purification and identification of linked proteins by mass spectrometry (Fig. 1A). Despite these additional functional groups, we were able to demonstrate that EET-P mimics 14,15-EET's previously reported ability to dilate mouse mesenteric arteries pre-constricted with thromboxane A2 agonist U46619 (13) (Fig. S1). We next used this probe to identify putative mVSMC 14,15-EET receptor(s) by treating mouse heart mVSMCs with EET-P ( $1 \mathrm{mM})$ in the presence or absence of 14,15-EET followed by a $5 \mathrm{~min}$ UV (365 nm) exposure. Cell lysates were reacted with biotin-azide for affinity purification and subsequent mass spectrometry analysis that yielded a number of intracellular and membrane-associated proteins that could be competitively displaced by 14,15-EET, including a known 14,15-EET metabolizing enzyme, epoxide hydrolase, an indication of probe specificity (Table S1; complete MS data has been deposited to the ProteomeXchange Consortium via the PRIDE database; dataset identifier PXD013952), A single GPCR was detected in the screen, GPR39, a $50 \mathrm{kDa}$ orphan member of the ghrelin receptor family previously reported to be activated by zinc ions $\left(\mathrm{Zn}^{2+}\right)(14)$. We also found that cultured VSMCs could be labeled with EET-P using a rhodamine azide in-cell click reaction. Importantly, this membrane labeling can be displaced by pretreatment with 14,15-EET (Fig. 1B). We further confirmed membrane-bound targets of EET-P by using rhodamine-azide labeling followed by membrane purification and SDS-PAGE analysis to detect EET-P-labeled proteins and observed a single band that was dose-dependently displaced by 14,15EET and exhibited approximately the same molecular weight as GPR39 (Fig 1C). The GPR39 genomic locus encodes two isoforms, GPR39 1a (a full-length 7-transmembrane (7TM) isoform) and 1b (a truncated 5TM 1b isoform that lacks TM6 and 7) (15). Using immunocytochemistry and real-time quantitative PCR we confirmed expression of GPR39 1a, but not 1b in cultured mouse heart mVSMCs (Fig. 1D). We also determined that human embryonic kidney (HEK)-293 cells express only the $1 \mathrm{~b}$ isoform (Fig. S2B). Probe binding specificity was confirmed by crosslinking EET-P to HEK-293 cells transiently transfected with HA-tagged human GPR39 1a (Fig. S2C-E). Western blots probed with HA-antisera (Fig. 1E) indicate that HA-tagged GRP39 1a co-purifies with EET-P (lane 1) and this band is absent in lysates of untransfected cells (lane 4). We further confirmed probe specificity by eliminating probe binding to HAtagged GPR39 by pretreating GPR39-transfected HEK-293 cells with either 14,15-EET ( 5 mM, lane 2) or 
EETs antagonist 14,15-epoxyeicosa-5(Z)-enoic acid (16) (14,15-EEZE, $5 \mathrm{mM}$, lane 3) prior to EET-P exposure. Finally, dot-blot assay demonstrated dose-dependent saturable binding of 14,15-EET, but not 11,12-EET, to protein extracts from HEK-293 cells expressing GPR39 1a, but not control cells (Fig 1F).

\section{Isoform-Specific Activation of GPR39 Signaling by 14,15-EET and 15-HETE}

We transfected HEK-293 cells with either GPR39 1a or GPR39 1b to determine if 14,15-EET binding activates GPR39 signaling and if 14,15-EET binding is specific or whether related eicosanoids can also activate this receptor. Cells were subsequently stimulated with one of four regioisomers of EETs (5,6-EET; 8,9-EET; 11,12-EET; 14,15-EET), HETEs (11-HETE, 12-HETE, 15-HETE, 20-HETE), or vehicle. 14,15-EET and 15-HETE are the only regioisomers that significantly increase GPCR activation as monitored by ERK phosphorylation in cells transfected with GPR39 1a (Fig. 2A, 2B) but not untransfected cells (Fig. 2C-D), with both eicosanoids displaying a concentration-dependent activation of GPR39 (Fig. 2C and 2D). Zinc has been reported to be either a GPR39 agonist (14) or an allosteric modulator for synthetic ligands of the receptor (17). Therefore, we repeated the dose response-curves for 14,15-EET and 15-HETE in the presence of $1 \mu \mathrm{M}$ zinc, which has no effect on ERK phosphorylation by itself. Zinc significantly potentiates the effects of both eicosanoids, consistent with its role as an allosteric modulator of GPR39. Dot-blot assays using lysates from GPR39 1a stably expressing HEK-293 cells demonstrate dosedependent, saturable binding of 15-HETE, similar to 14,15-EET, that is not observed for 12-HETE (Fig S3A). Importantly, binding competition experiments indicate that 15-HETE and 14,15-EET can displace each other while 12-HETE (Fig S3B) and 11,12-EET (Fig S3C), respectively, fail to do so.

\section{GPR39-Ligand Interaction Modeling Predicts Selectivity for 14,15-EET and 15-HETE}

We next used a homology model to investigate GPR39 1a-ligand specificity in silico (Fig S4A and B) that predicted structural complementarity between 14,15-EET and GPR39, as indicated by a low docking energy $(-8.7 \mathrm{kcal} / \mathrm{mol})$. A fingerprint similarity search for other GPR39 ligands using 14,15-EET as a reference structure resulted in a list of long-chain fatty acids (Table S2) including 15-HETE, which had a predicted docking energy of $-8.6 \mathrm{kcal} / \mathrm{mol}$, similar to 14,15 -EET. This likely reflects that both compounds have a polar group at the $15^{\text {th }}$ carbon position and maintain double bonds in the cis configuration at the $5^{\text {th }}, 8^{\text {th }}$ and $11^{\text {th }}$ positions (Fig. 2E) unlike the other EETs and HETEs regioisomers. A potential orthostatic pocket was identified using SiteMap V3.2 which revealed a hydrophilic major pocket and a hydrophobic minor pocket (Fig S4C) in GPR39 1a with the tail portion of these lipids extending into the minor binding pocket formed by transmembrane 1 (TM1), TM2, TM7 and the N-terminal loop (Fig. 2F, S4D-E). The major pocket accommodates the carboxylate moiety of 14,15-EET and 15-HETE by forming ionic interactions with positively charged residues from TM6 (Fig. 2F).

\section{GPR39 Mediates Effects of 14,15-EET and 15-HETE on mVSMC Calcium Transients}

We next enquired whether 14,15-EET and 15-HETE regulate the signaling of mVSMC via GPR39, and if their actions are modulated by zinc. Using live-cell fluorescent imaging to monitor calcium transients, we first evaluated the effect of $\mathrm{Zn}^{2+}$ alone in mVSMCs and found that it increases intracellular calcium with 
an EC50 of $7.8 \mathrm{mM}$ (Fig 3A). We then established the dose-response relationship for 15-HETE in the presence and absence of 1, 4 and $10 \mathrm{mM}$ zinc. We observed that zinc, at 4 or $10 \mathrm{mM}$, significantly potentiates the response of GPR39 to 15-HETE stimulation (Fig 3B; only 1 and $10 \mu \mathrm{M}$ are shown). Application of 15-HETE alone elicits a calcium response in mVSMCs only at concentrations above 100 nM (Fig. 3B). However, in the presence of $10 \mu \mathrm{M}$ (Fig. 3B) or $4 \mu \mathrm{M}$ (Fig. 3C) zinc, 15-HETE elicits a significant calcium response in mVSMCs at low nanomolar concentrations (Fig. 3B), suggesting that zinc serves as a positive allosteric modulator for 15-HETE in mVSMCs. Importantly, GPR39 RNAi knockdown in mVSMCs attenuated the increase in mVSMC intracellular calcium produced by 15 -HETE (Fig 3C, S5B), confirming a significant role for GPR39 in mediating the effect of 15-HETE on mVSMC calcium transients. 14,15-EET had no effect on intracellular calcium in mVSMCs at concentrations between $1 \mathrm{pM}$ $10 \mathrm{mM}$ (data not shown), but it was observed to inhibit 15-HETE-dependent increases in intracellular calcium (Fig. 3D), consistent with GPR39 acting as a dual sensor for both 14,15-EET and 15-HETE. Taken together, our modeling data along with pERK, calcium imaging and binding assays indicate a competitive structure-activity relationship between 14,15-EET and 15-HETE.

\section{GPR39 Localization and Function in Myocardial Microvessels}

Consistent with a role in regulating coronary vascular resistance, immunofluorescence indicates that GPR39 expression is predominantly restricted to microvessels in the mouse heart (Fig 4A). Co-staining with a-smooth muscle actin (a-SMA, upper panel) and CD31 (middle panel) confirms expression in arteriolar smooth muscle cells, but not endothelial cells (Fig 4A). Antibody specificity was confirmed using heart tissue from GPR39 knockout (KO) mice, which lacked microvascular immunoreactivity observed in hearts from wild-type littermates (Fig 4A, lower panel). This microvascular pattern of expression was further confirmed using non-fluorescent immunostaining where we also observed GPR39 expression in both mouse and human heart within perivascular cells that were consistently adjacent to microvessels including capillaries (Fig. S6), suggestive of GPR39 expression in pericytes as well.

\section{Role of GPR39 in Microvascular Regulation and Response to Myocardial Ischemia}

We next determined the contribution of GPR39 activation by eicosanoids to microvascular tone regulation. This was accomplished using a mouse heart Langendorff preparation where changes in coronary perfusion pressure (CPP) are determined by microvascular resistance at a constant flow rate. Accordingly, 15-HETE (1 $\mu \mathrm{M})$ increases CPP (Fig 4B), and this effect is inhibited by co-administration of 14,15-EET $(1 \mu \mathrm{M})$ or abolished in GPR39-null hearts (Fig 4B, Fig S7). Importantly, the effects of two other vasoactive agents on CPP, angiotensin II (Ang II) and prostaglandin $\mathrm{I}_{2}\left(\mathrm{PGI}_{2}\right)$, were unaffected by GPR39 deletion. Interestingly, CPP response to sodium nitroprusside (SNP) was significantly attenuated in GPR39 KO. Finally, consistent with the established role of P450 eicosanoids in myocardial ischemia, GPR39 KO mice were protected from I/R injury in-vivo compared to their wild-type littermates (Fig 4C).

\section{Discussion}


We developed a chemical crosslinking mimetic to identify 14,15 -EET as an endogenous ligand of GPR39, canonically consider to be a zinc receptor. We further show that 14,15-EET and 15-HETE can regulate mVSMC calcium dynamics and coronary vascular resistance via their opposing actions on GPR39. We also provide in vivo evidence that GPR39 contributes to I/R injury in the heart, suggesting that GPR39 may serve as a therapeutic target in myocardial ischemia.

Models of 14,15-EET signaling mediated by a GPCR have been proposed by several groups $(13,18,19)$ yet the receptor has remained unidentified. Photoaffinity labeling was previously used to demonstrate a 47 kDa high-affinity binding protein of unknown identity for 14,15-EET in membrane fractions from U937 and vascular cells (13). Unlike previous approaches, our clickable photocrosslinking probe allowed enrichment of crosslinked proteins from mVSMCs, permitting target identification by mass spectrometry. GPR39 represents the first high-affinity receptor for any EET regioisomer, although several membrane proteins have been shown to respond to high micromolar concentrations of 14,15-EET including: large conductance calcium-activated (BKCa) and ATP-sensitive potassium channels; , the transient receptor potential cation channel subfamily V member 4 (TRPV4); and some GPCRs (13), including GPR40 (20) and the prostaglandin E (EP2) (18) and thromboxane (TP) (19) receptors; as well as our own recent work screening a variety of GPCRs for 14,15-EET responses (21). Our modeling predictions also led us to test and confirm that 15-HETE is an additional endogenous ligand of GPR39. No high affinity receptor has been identified for 15-HETE, although receptors have been identified for other HETE regioisomers, including 12-HETE (22) and 20-HETE (23).

Endogenous ligands for GPR39 have remained elusive as early reports suggested obestatin, a ghrelinderived peptide, but this has since been refuted $(14,24)$. Multiple studies have linked GPR39 with metabolism including two studies of GPR39 KO mice, one where body weight and food intake were reported to be normal ((25)), while the other noted higher body weight and fat composition with no change in food intake (26). A report has also linked GPR39 loss with impaired insulin secretion (27), yet synthetic agonists of GPR39 fail to drive insulin secretion (28), and still others associate GPR39 loss with nervous system symptoms such as seizures (29) and depression-like behavior (30). GPR39 has also been proposed to function as a $\mathrm{Zn}^{2+}$ receptor ((14)). However, it is unclear if the pharmacokinetics of $\mathrm{Zn}^{2+}$ with GPR39 support it as a physiological agonist of the receptor. It is more likely that zinc simply modulates receptor activity based on studies characterizing synthetic GPR39 agonists (17). Furthermore, $\mathrm{Zn}^{2+}$ is known to be an allosteric modulator for multiple receptors and ion channels (31). Here, we confirm GPR39 sensitivity to high concentrations of $\mathrm{Zn}^{2+}$, and critically provide a novel function for physiologic levels of $\mathrm{Zn}^{2+}$ as an allosteric modulator enhancing the efficacy of the natural GPR39 ligands 14,15-EET and 15-HETE. Thus, $\mathrm{Zn}^{2+}$ may also play a role in the regulation and disorders of the microcirculation through GPR39 modulation. Recent reports suggested that zinc regulates endothelial function (32) and inhibits phosphate-induced vascular calcification (33), presumably through its action on GPR39. 
The concentrations of 14,15-EET, 15-HETE and zinc used in our study are similar to levels measured in human blood under physiological and pathophysiological conditions. The concentration of 15-HETE in normal plasma ranges from $0.8+/-0.023 \mathrm{nM}$ (34) to $0.1 \mathrm{uM}$ (35). Higher levels have been measured in serum (36-38). Importantly, 15-HETE levels are increased in patients with chronic inflammatory conditions (39) and vascular diseases, including coronary artery disease (40) and pulmonary arterial hypertension $(41,42)$. Similarly, the concentration of EETs in plasma from healthy volunteers was reported to be in the low nanomolar range (median of $0.6 \mathrm{nM}$ ), with coronary artery disease (CAD) patients having higher EETs (median $1 \mathrm{nM}$ ) (43). Among patients with CAD, plasma EETs were inversely related to disease severity, with lower EETs found in patients with obstructive compared to non-obstructive CAD or to patients with no apparent CAD (44). Finally, levels of eicosanoids are even higher in heart tissues compared to plasma (40), likely reaching effective concentrations at the receptor level. Mean serum zinc concentration is around $10 \mu \mathrm{M}$, with lower levels in young children, peak concentrations in young adults and a slow decline with age (45). Zinc is present in all body tissues and fluids. Plasma zinc is only about 0.1 percent of total body zinc content, with approximately $30 \%$ of total body zinc content in skeletal muscle (46).

In conclusion, our study is the first to propose a role for GPR39 in microvascular regulation and myocardial ischemia, in part by sensing the relative concentrations of 14,15-EET and 15-HETE. It is possible that the imbalance between these two eicosanoids could alter GPR39 activity, contributing to myocardial ischemia, and predisposing individuals to microvascular complications of systemic diseases such as diabetes, hypertension and septic shock. Work by numerous investigators over the past three decades has established a critical role for P450 eicosanoids in cardiovascular physiology and disease (47-52). Further progress in this field has been hampered by the lack of understanding of the molecular mechanisms of actions of these important lipid mediators. Our identification of the receptor for two important endogenous vasoactive eicosanoids and their unique mode of dual and opposing regulation of receptor function is a major breakthrough in the field. These findings will have a significant impact on our understanding of how P450 eicosanoids control the microcirculation, their roles in microvascular disease, and the development of novel therapeutic agents for vascular disease.

\section{Declarations}

This study was conducted in accordance with the National Institutes of Health guidelines for the care and use of animals in research, and protocols were approved by the Institutional Animal Care and Use Committee at Oregon Health and Science University, Portland, OR, USA

Acknowledgments: Studies were supported by the Knight Cardiovascular Institute. Mass spectrometric analysis was performed by the OHSU Proteomics Shared Resource with partial support from NIH core grants P30EY010572, P30CA069533, and shared instrumentation grant S100D012246.

\section{References}


1. J. M. Muller, M. J. Davis, W. M. Chilian, Integrated regulation of pressure and flow in the coronary microcirculation. Cardiovasc Res 32, 668-678 (1996).

2. J. H. Capdevila, J. R. Falck, R. C. Harris, Cytochrome P450 and arachidonic acid bioactivation. Molecular and functional properties of the arachidonate monooxygenase. J Lipid Res 41, 163-181 (2000).

3. R. J. Roman, P-450 metabolites of arachidonic acid in the control of cardiovascular function. Physiol Rev 82, 131-185 (2002).

4. W. B. Campbell, D. Gebremedhin, P. F. Pratt, D. R. Harder, Identification of epoxyeicosatrienoic acids as endothelium-derived hyperpolarizing factors. Circ Res 78, 415-423 (1996).

5. B. Fisslthaler et al., Cytochrome P450 2C is an EDHF synthase in coronary arteries. Nature 401, 493497 (1999).

6. D. R. Harder, N. J. Alkayed, A. R. Lange, D. Gebremedhin, R. J. Roman, Functional hyperemia in the brain: hypothesis for astrocyte-derived vasodilator metabolites. Stroke 29, 229-234 (1998).

7. X. Peng et al., Suppression of cortical functional hyperemia to vibrissal stimulation in the rat by epoxygenase inhibitors. Am J Physiol Heart Circ Physiol 283, H2029-2037 (2002).

8. J. J. Iliff et al., Epoxyeicosanoid signaling in CNS function and disease. Prostaglandins Other Lipid Mediat 91, 68-84 (2010).

9. D. Gebremedhin, S. Gopalakrishnan, D. R. Harder, Endogenous events modulating myogenic regulation of cerebrovascular function. Curr Vasc Pharmacol 12, 810-817 (2014).

10. A. P. Zou et al., Inhibition of renal vascular 20-HETE production impairs autoregulation of renal blood flow. Am J Physio/ 266, F275-282 (1994).

11. S. L. Hoopes, V. Garcia, M. L. Edin, M. L. Schwartzman, D. C. Zeldin, Vascular actions of 20-HETE. Prostaglandins Other Lipid Mediat 120, 9-16 (2015).

12. P. L. Li, W. B. Campbell, Epoxyeicosatrienoic acids activate $\mathrm{K}+$ channels in coronary smooth muscle through a guanine nucleotide binding protein. Circ Res 80, 877-884 (1997).

13. Y. Chen, J. R. Falck, V. L. Manthati, J. L. Jat, W. B. Campbell, 20-lodo-14,15-epoxyeicosa-8(Z)-enoyl-3azidophenylsulfonamide: photoaffinity labeling of a 14,15-epoxyeicosatrienoic acid receptor. Biochemistry 50, 3840-3848 (2011).

14. B. Holst et al., GPR39 signaling is stimulated by zinc ions but not by obestatin. Endocrinology 148, 13-20 (2007).

15. K. L. Egerod et al., GPR39 splice variants versus antisense gene LYPD1: expression and regulation in gastrointestinal tract, endocrine pancreas, liver, and white adipose tissue. Mol Endocrino/ 21, 16851698 (2007).

16. K. M. Gauthier et al., 14,15-Epoxyeicosa-5(Z)-enoic acid: a selective epoxyeicosatrienoic acid antagonist that inhibits endothelium-dependent hyperpolarization and relaxation in coronary arteries. Circ Res 90, 1028-1036 (2002). 
17. S. Sato, X. P. Huang, W. K. Kroeze, B. L. Roth, Discovery and Characterization of Novel GPR39 Agonists Allosterically Modulated by Zinc. Mol Pharmacol 90, 726-737 (2016).

18. C. Yang et al., 14,15-Epoxyeicosatrienoic acid induces vasorelaxation through the prostaglandin EP(2) receptors in rat mesenteric artery. Prostaglandins Other Lipid Mediat 93, 44-51 (2010).

19. D. J. Behm, A. Ogbonna, C. Wu, C. L. Burns-Kurtis, S. A. Douglas, Epoxyeicosatrienoic acids function as selective, endogenous antagonists of native thromboxane receptors: identification of a novel mechanism of vasodilation. J Pharmacol Exp Ther 328, 231-239 (2009).

20. S. K. Ma et al., Overexpression of G-protein-coupled receptor 40 enhances the mitogenic response to epoxyeicosatrienoic acids. PLoS One 10, e0113130 (2015).

21. X. Liu et al., Functional screening for G protein-coupled receptor targets of 14,15-epoxyeicosatrienoic acid. Prostaglandins Other Lipid Mediat 132, 31-40 (2017).

22. Y. Guo et al., Identification of the orphan G protein-coupled receptor GPR31 as a receptor for 12-(S)hydroxyeicosatetraenoic acid. J Biol Chem 286, 33832-33840 (2011).

23. V. Garcia et al., 20-HETE Signals Through G-Protein-Coupled Receptor GPR75 (Gq) to Affect Vascular Function and Trigger Hypertension. Circ Res 120, 1776-1788 (2017).

24. E. Lauwers, B. Landuyt, L. Arckens, L. Schoofs, W. Luyten, Obestatin does not activate orphan G protein-coupled receptor GPR39. Biochem Biophys Res Commun 351, 21-25 (2006).

25. F. Tremblay et al., Normal food intake and body weight in mice lacking the G protein-coupled receptor GPR39. Endocrinology 148, 501-506 (2007).

26. D. Moechars et al., Altered gastrointestinal and metabolic function in the GPR39-obestatin receptorknockout mouse. Gastroenterology 131, 1131-1141 (2006).

27. B. Holst et al., G protein-coupled receptor 39 deficiency is associated with pancreatic islet dysfunction. Endocrinology 150, 2577-2585 (2009).

28. O. Fjellstrom et al., Novel Zn2+ Modulated GPR39 Receptor Agonists Do Not Drive Acute Insulin Secretion in Rodents. PLoS One 10, e0145849 (2015).

29. D. Gilad et al., Homeostatic regulation of KCC2 activity by the zinc receptor mZnR/GPR39 during seizures. Neurobiol Dis 81, 4-13 (2015).

30. K. Mlyniec, B. Budziszewska, B. Holst, B. Ostachowicz, G. Nowak, GPR39 (zinc receptor) knockout mice exhibit depression-like behavior and CREB/BDNF down-regulation in the hippocampus. Int J Neuropsychopharmacol 18, (2014).

31. F. A. Peralta, J. P. Huidobro-Toro, Zinc as Allosteric Ion Channel Modulator: Ionotropic Receptors as Metalloproteins. Int J Mol Sci 17, (2016).

32. D. Zhu et al., Zinc regulates vascular endothelial cell activity through zinc-sensing receptor ZnR/GPR39. Am J Physiol Cell Physiol 314, C404-C414 (2018).

33. J. Voelkl et al., Zinc Inhibits Phosphate-Induced Vascular Calcification through TNFAIP3-Mediated Suppression of NF-kappaB. J Am Soc Nephrol, (2018). 
34. O. Quehenberger et al., Lipidomics reveals a remarkable diversity of lipids in human plasma. J Lipid Res 51, 3299-3305 (2010).

35. R. W. Walenga, S. Boone, M. J. Stuart, Analysis of blood HETE levels by selected ion monitoring with ricinoleic acid as the internal standard. Prostaglandins 34, 733-748 (1987).

36. L. L. Mazaleuskaya et al., Analysis of HETEs in human whole blood by chiral UHPLC-ECAPCI/HRMS. J Lipid Res 59, 564-575 (2018).

37. N. Psychogios et al., The human serum metabolome. PLoS One 6, e16957 (2011).

38. R. W. Walenga, S. Sunderji, M. J. Stuart, Formation of hydroxyeicosatetraenoic acids (HETE) in blood from adults versus neonates: reduced production of 12-HETE in cord blood. Pediatr Res 24, 563-567 (1988).

39. S. Lu et al., Utility of 15(S)-HETE as a Serological Marker for Eosinophilic Esophagitis. Sci Rep 8, 14498 (2018).

40. A. Lundqvist et al., The Arachidonate 15-Lipoxygenase Enzyme Product 15-HETE Is Present in Heart Tissue from Patients with Ischemic Heart Disease and Enhances Clot Formation. PLoS One 11, e0161629 (2016).

41. N. Al-Naamani et al., Plasma 12- and 15-hydroxyeicosanoids are predictors of survival in pulmonary arterial hypertension. Pulm Circ 6, 224-233 (2016).

42. M. H. Shishehbor et al., Systemic elevations of free radical oxidation products of arachidonic acid are associated with angiographic evidence of coronary artery disease. Free Radic Biol Med 41, 16781683 (2006).

43. K. N. Theken et al., Evaluation of cytochrome P450-derived eicosanoids in humans with stable atherosclerotic cardiovascular disease. Atherosclerosis 222, 530-536 (2012).

44. A. Oni-Orisan et al., Cytochrome P450-derived epoxyeicosatrienoic acids and coronary artery disease in humans: a targeted metabolomics study. J Lipid Res 57, 109-119 (2016).

45. C. Hotz, J. M. Peerson, K. H. Brown, Suggested lower cutoffs of serum zinc concentrations for assessing zinc status: reanalysis of the second National Health and Nutrition Examination Survey data (1976-1980). Am J Clin Nutr 78, 756-764 (2003).

46. Human Vitamin and Mineral Requirements: Report of a Joint FAO/WHO Expert Consultation. (2002).

47. W. Zhang et al., Soluble epoxide hydrolase: a novel therapeutic target in stroke. J Cereb Blood Flow Metab 27, 1931-1940 (2007).

48. D. A. Siler et al., Protective role of p450 epoxyeicosanoids in subarachnoid hemorrhage. Neurocrit Care 22, 306-319 (2015).

49. J. Seubert et al., Enhanced postischemic functional recovery in CYP2J2 transgenic hearts involves mitochondrial ATP-sensitive K+ channels and p42/p44 MAPK pathway. Circ Res 95, 506-514 (2004).

50. M. Renic et al., Effect of 20-HETE inhibition on infarct volume and cerebral blood flow after transient middle cerebral artery occlusion. J Cereb Blood Flow Metab 29, 629-639 (2009). 
51. K. Node et al., Anti-inflammatory properties of cytochrome P450 epoxygenase-derived eicosanoids. Science 285, 1276-1279 (1999).

52. E. A. Crago et al., Cerebrospinal fluid 20-HETE is associated with delayed cerebral ischemia and poor outcomes after aneurysmal subarachnoid hemorrhage. Stroke 42, 1872-1877 (2011).

\section{Figures}

A
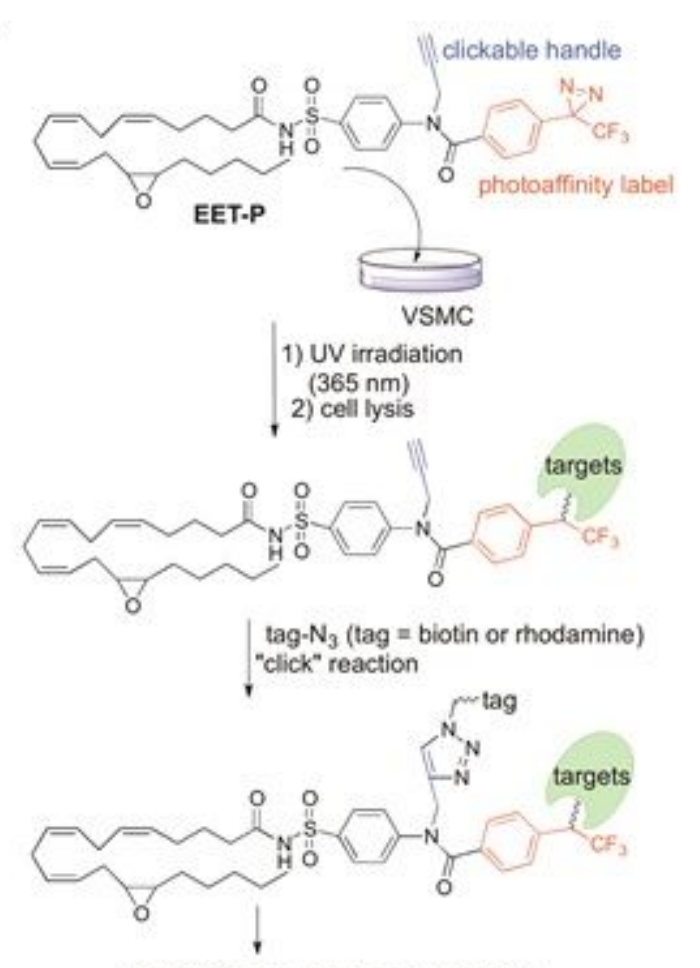

SDS-PAGE or imaging or enrichment

B

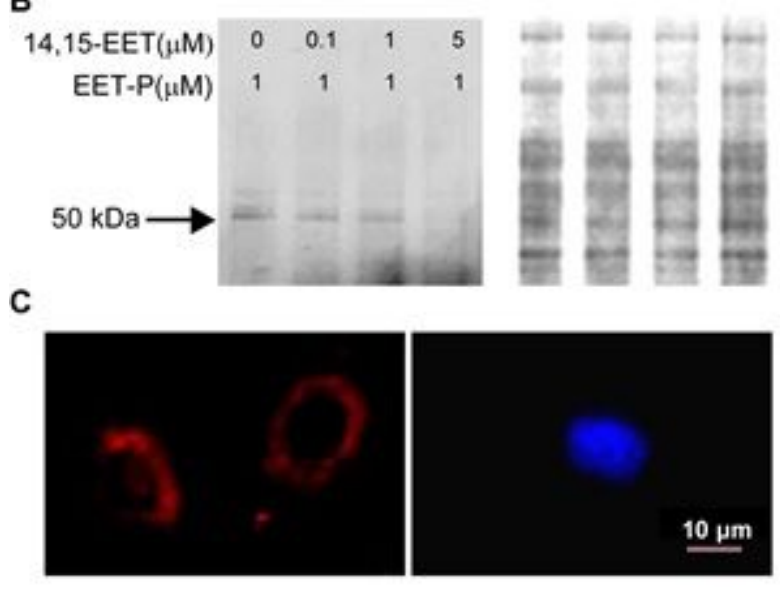

D
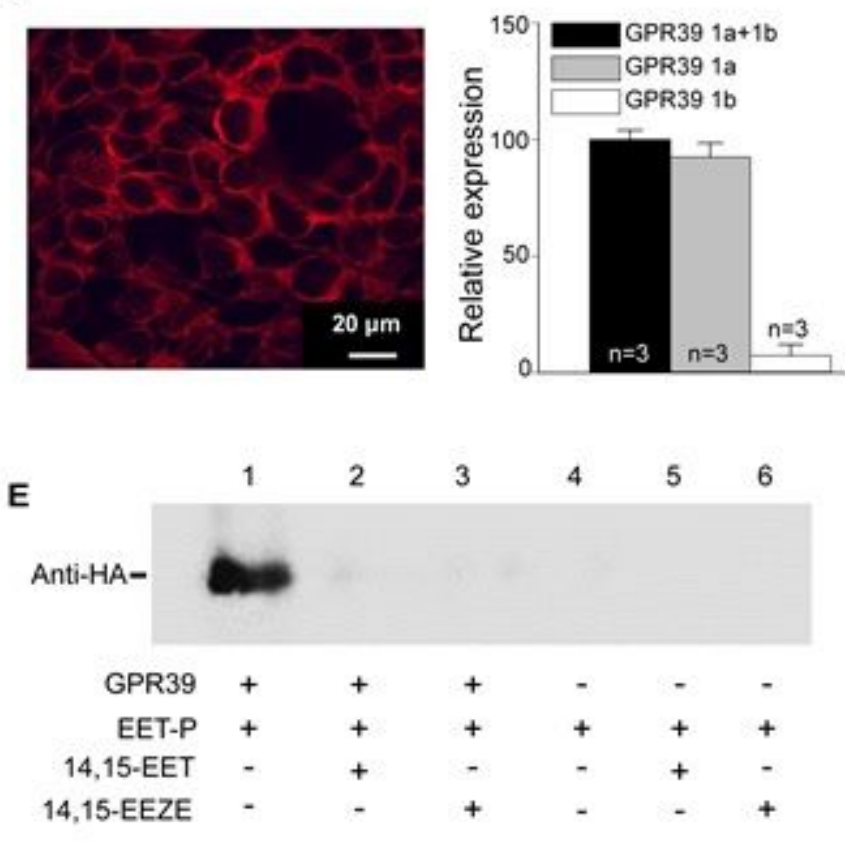

$\mathbf{F}$

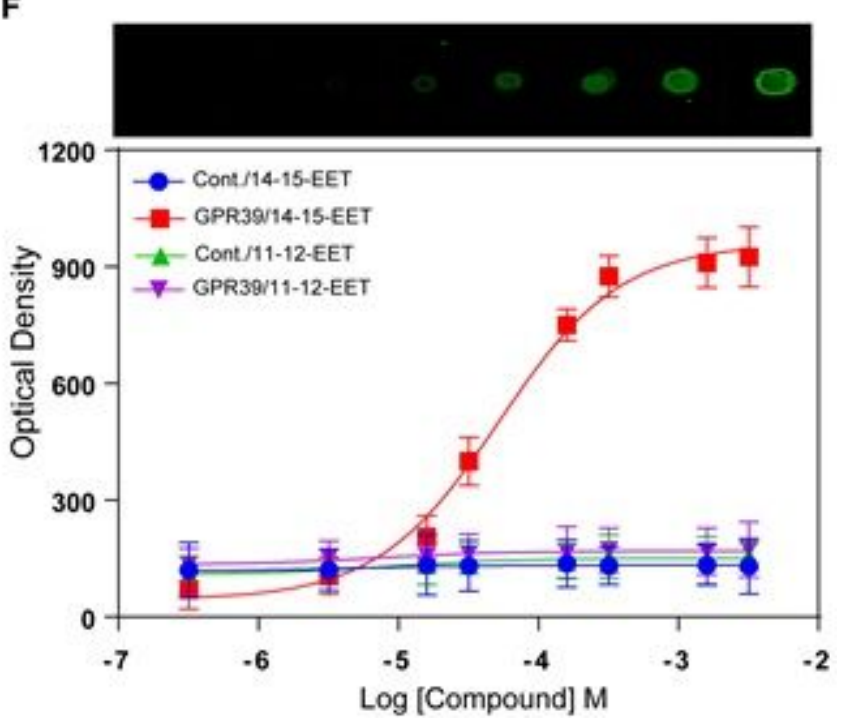

\section{Figure 1}

Purification and validation of GPR39 as a putative receptor for 14,15-EET. (A) Chemoproteomics strategy to identify 14,15-EET receptor in mVSMCs. (B) Photocrosslinking GPR39 with EET-P in mVSMCs. Left: SDS-PAGE gel revealed the presence of a $\sim 50 \mathrm{kDa}$ band. 14,15-EET pre-treatment reduced protein binding to EET-P in a dose-dependent manner. Right: Total protein determined by Coomassie Blue staining of the 
same gel. (C) Confocal images demonstrating binding of EET-P to outer surface of mVSMCs (left, red). Pretreatment with $1 \mu \mathrm{M}$ 14,15-EET for 10 min prevented EET-P surface binding (right; blue color is nuclear stain DAPI). (D) A representative immunofluorescent confocal image illustrating GPR39 expression (left, red) in primary cultured mouse heart mVSMCs (scale bar $=20 \mu \mathrm{m}$ ), and expression of GPR39 1a, but not $1 \mathrm{~b}$, confirmed by qPCR (right). (E) Western blot detection of EET-P crosslinking to epitope-tagged GPR39 1a in transfected HEK cells. HEK cells were treated with $5 \mu \mathrm{M} 14,15$-EET or 14,15-EEZE for 10 min, $1 \mu \mathrm{M}$ EET-P for $15 \mathrm{~min}$, and then irradiated with UV for $5 \mathrm{~min}$ at $4 \mathrm{oC}$. After clicking with biotin and purification with streptavidin Dynabeads, protein extracts were probed by anti-HA antibody. (F) Dot-blot assay illustrating dose-dependent binding of 14,15-EET, but not 11,12-EET, to membrane protein extracts from HEK-293 cells stably expressing GPR39 1a, but not untransfected control cells (raw images on top, and optical density quantification at the bottom; $n=3$ ).
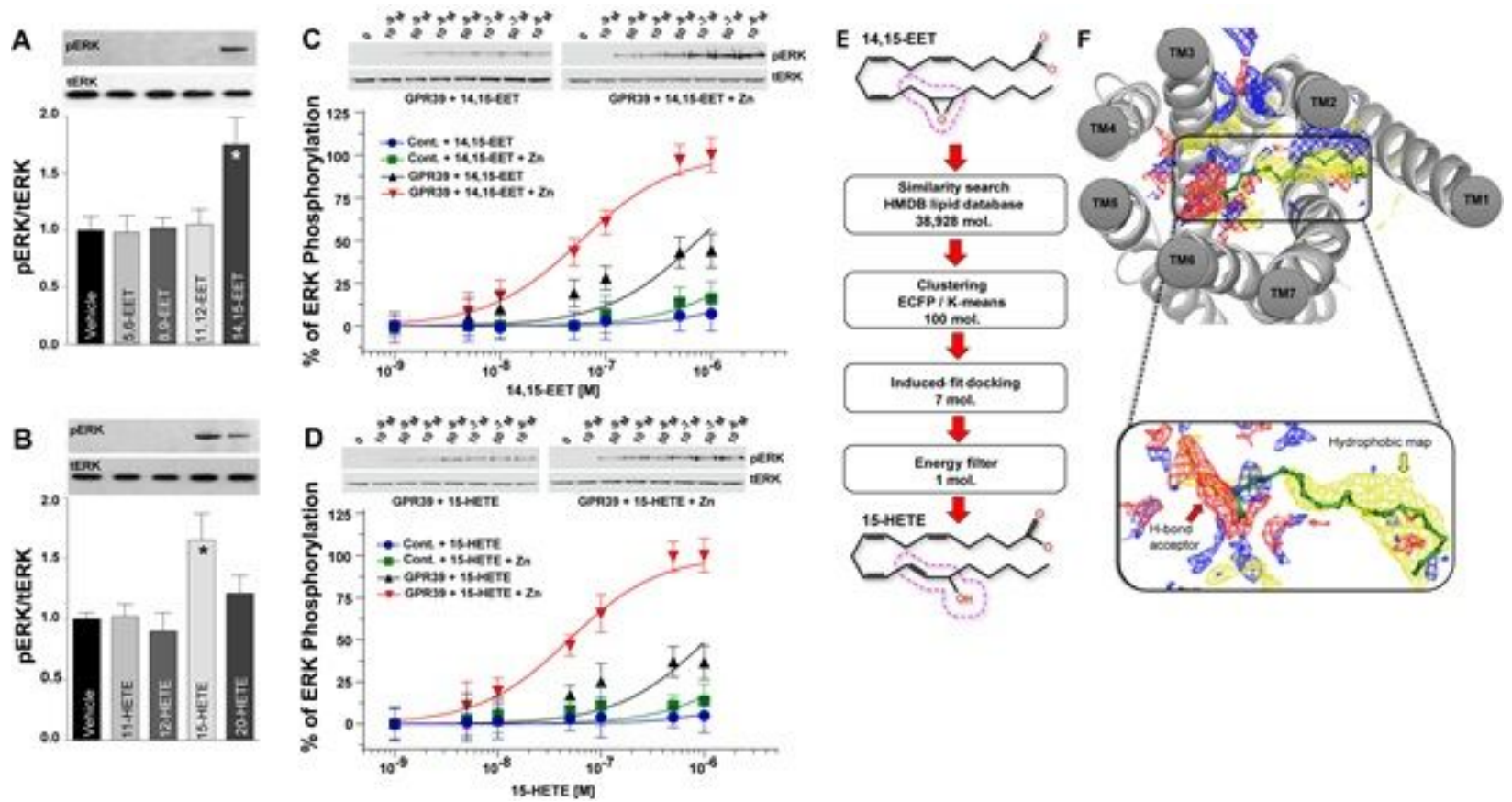

Figure 2

Functional activation and modeling of GPR39 ligand binding. ERK phosphorylation induced by 1 min treatment with $1 \mu \mathrm{M}$ of each one of 4 regioisomers of EETs $(A ; n=3)$ or HETEs $(B ; n=3)$. Total ERK was used as a protein loading control. Dose-dependent ERK phosphorylation induced by 1 min treatment with either 14,15-EET ( $C ; n=3)$ or 15-HETE ( $D ; n=3)$ in HEK cells expressing GPR39 1a, but not untransfected cells, in the presence and absence of $1 \mu \mathrm{M}$ zinc. (E) Virtual Screening of 14,15-EET-like compounds from HMDB database revealed comparable energies for 15-HETE and 14,15-EET. The 14,15-EET and 15-HETE structures exhibit a high degree of structural similarity. The dotted red line around carbons 13-15 highlights the structural difference between the two eicosanoids. (F) GPR39 7TM core binding pocket and an enlarged view of the predicted binding pose of 14,15-EET. Blue, red and yellow maps correspond to hydrogen bond donor, hydrogen bond acceptor and hydrophobic areas within the binding pocket, respectively. $\mathrm{N}$-terminal residues contributing to minor pocket are not shown for the sake of clarity. The 
carboxylate group of 14,15-EET interacts with polar residues in TM6 and the lipid portion interacts with the hydrophobic site formed at the minor-binding pocket, as shown in site mapping.
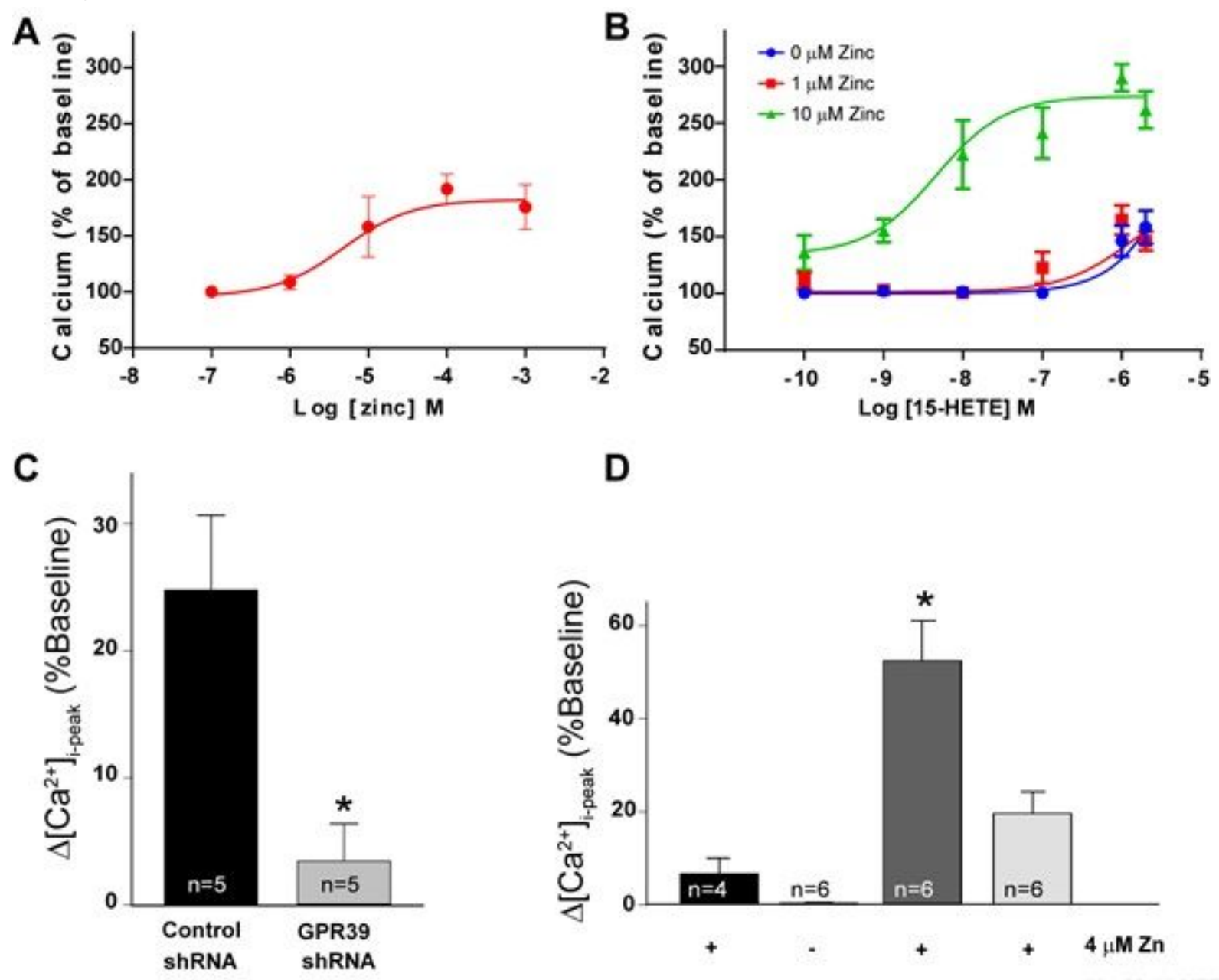

D

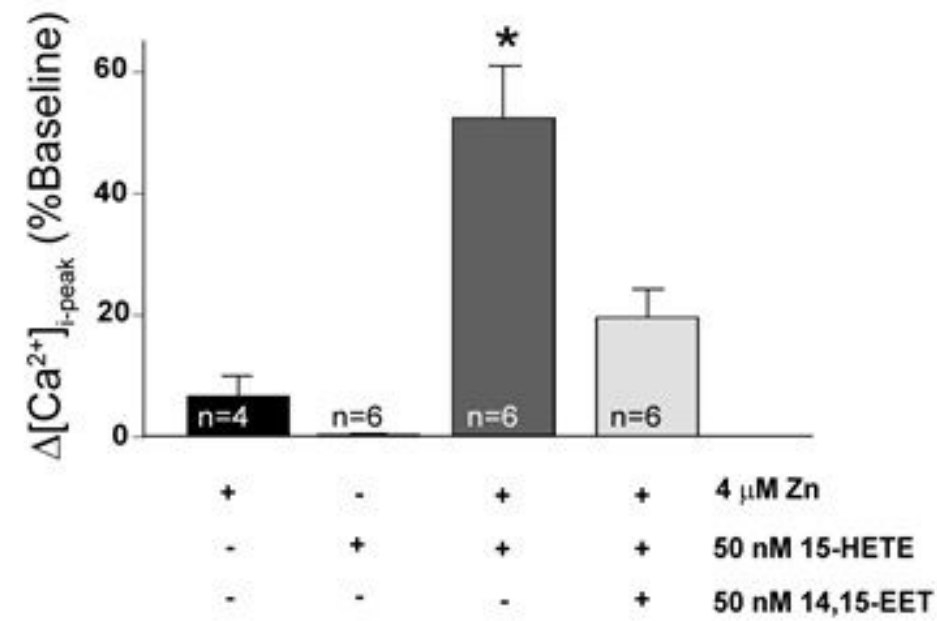

Figure 3

(A) Concentration-dependent increase in [Ca2+]i in mVSMCs in response to extracellular zinc $(n=5$ for each concertation). (B) Dose-dependent increase in mVSMC [Ca2+]i in response to 15-HETE with and without zinc ( $n=5-7)$. (C) Summary of [Ca2+]i response to $1 \mu \mathrm{M} 15$-HETE in mVSMCs treated with a lentivirus containing either a scrambled or GPR39-targetting shRNA for 72 hours $(n=5)$. (D) Summary of changes in mVSMCs [Ca2+]i in response to $50 \mathrm{nM}$ of 14,15-EET and 15-HETE, separately and in combination, and with and without zinc $(n=4-6)$. When applied alone, neither $4 \mu \mathrm{M}$ zinc nor 50 nM 15HETE had an effect on [Ca2+]i. Zinc $(4 \mu \mathrm{M})$ potentiates the effect of 15-HETE (50 nM) on [Ca2+]i in mVSMCs, and the increase in mVSMCs [Ca2+]i by 15-HETE is abolished by pre-treatment with 14,15-EET. 

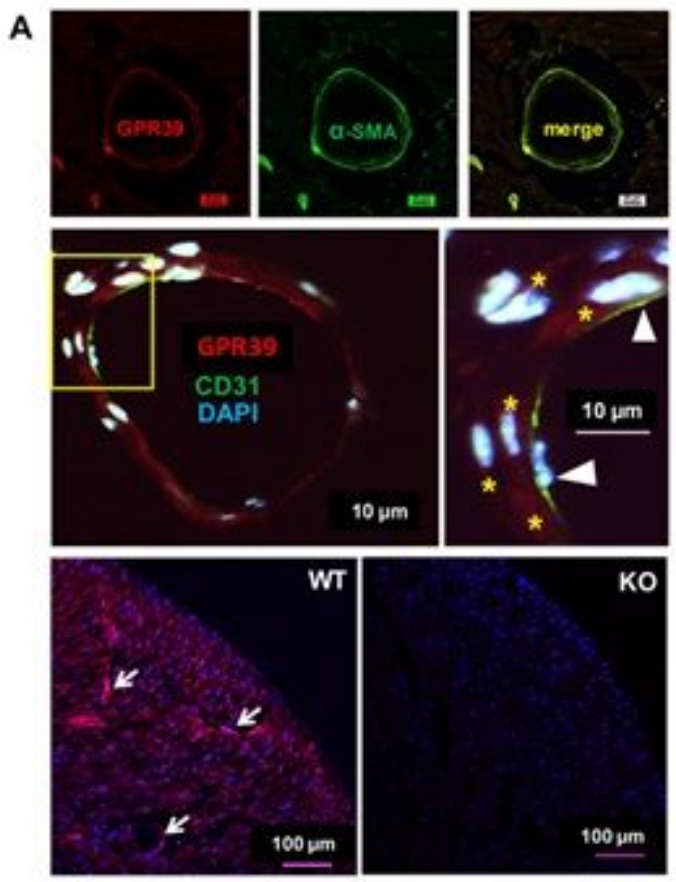

wT

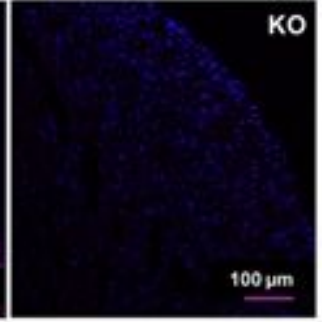

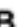

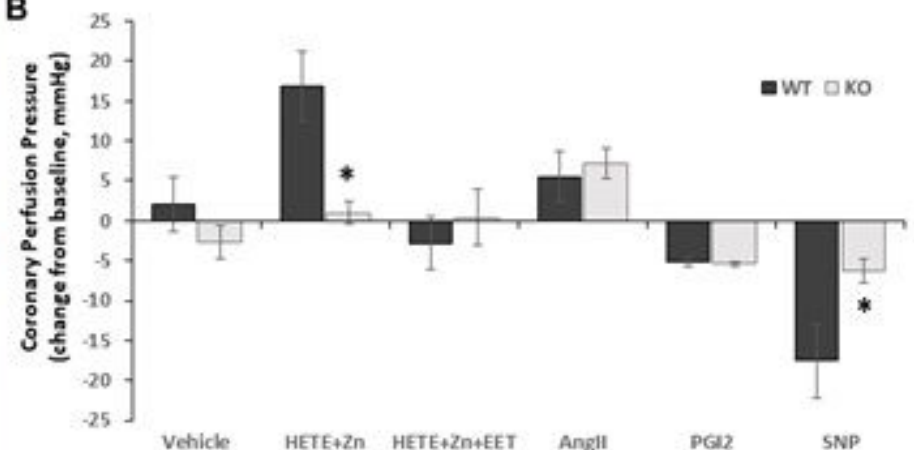

C
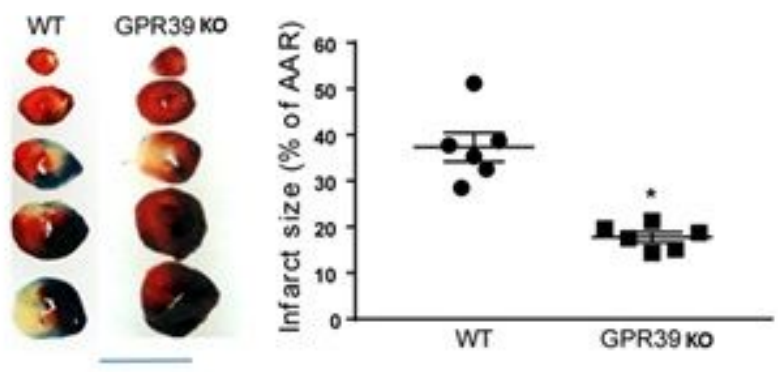

Figure 4

Microvascular localization and function of GPR39 in mouse heart. (A) Immunofluorescent imaging of GPR39 shows microvascular pattern of expression in mouse heart (upper left, red; scale bar $=20 \mu \mathrm{m}$ ), which co-localizes with VSMC markers a-smooth muscle actin (a-SMA, green, upper middle and right; scale bar $=20 \mu \mathrm{m}$ ), but not endothelial marker CD31 (middle panel; green and arrow heads in magnified view; yellow stars point to GPR39-positive mVSMCs within vascular wall in red; blue is DAPI nuclear staining; scale bar $=10 \mu \mathrm{m})$. The lower panel shows GPR39 immunoreactivity in wild-type (WT, left; arrows point to immunoreactive microvessels in red) and GPR39 knockout (KO; blue is nuclear stain DAPI) mouse heart tissue sections (scale bar $=100 \mu \mathrm{m}$ ). (B) Changes in coronary perfusion pressure, at a constant flow rate, in response to infusion of 15-HETE (1 $\mu \mathrm{M}$; in presence of $10 \mu \mathrm{M}$ zinc), 15-HETE plus 14,15-EET ( $1 \mu \mathrm{M}$; in presence of $10 \mu \mathrm{M}$ zinc), prostaglandin I2 (PGI2, $200 \mathrm{nM})$, angiotensin II (Angll, 100 $\mathrm{nM})$, sodium nitroprusside (SNP; $10 \mu \mathrm{M}$ ) or vehicle in isolated mouse heart preparation from WT and GPR39 KO mice ( $n=5)$. (C) Infarct size after myocardial ischemia/reperfusion (I/R) in WT and GPR39 KO mice. The left panel shows TTC-stained heart slices (scale bar in $1 \mathrm{~cm}$ ), and the right panel is a summary graph of infarct size in in WT and GPR39 KO mice $(n=6)$.

\section{Supplementary Files}

This is a list of supplementary files associated with this preprint. Click to download.

- SupplementalFigures.pdf

- SupplementaryMaterialFinal.docx 